\title{
Understanding the Solvent Contribution to Chemical Reaction Barriers
}

William Morris, ${ }^{\dagger}, \S$ Edward D. Lorance, ${ }^{*},{ }^{\dagger}$ and Ian R. Gould ${ }^{*}, \dagger$

† School of Molecular Sciences, Arizona State University, Tempe, Arizona 85287, United States

¥Department of Chemistry, Vanguard University, Costa Mesa, California 92626, United States

Supporting Information

page

Figure S1. Eyring Activation Enthalpy versus Pakar factor .......................................S2

Figure S2. Eyring Activation Entropy versus Pakar factor...........................................S3

Figure S3. Eyring Activation Enthalpy versus Born factor ...........................................S4

Figure S4. Eyring Activation Entropy versus Born factor ……………………..........S5

Figure S5. Eyring Activation Enthalpy versus Eyring Activation Entropy ..................S6 


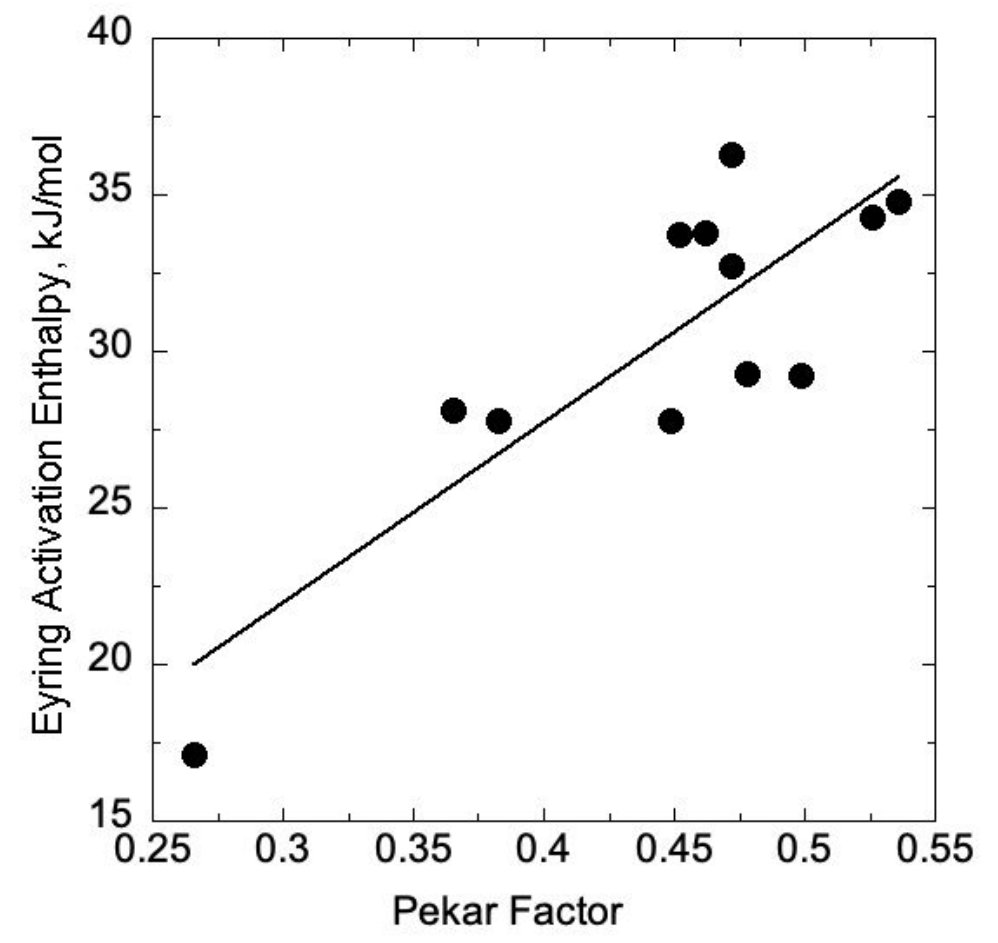

Figure S1. Plot of Eyring activation enthalpy for fragmentation of the pinacol radical cation as a function of the Pekar factor in various solvents. The correlation coefficient is 0.842 . 


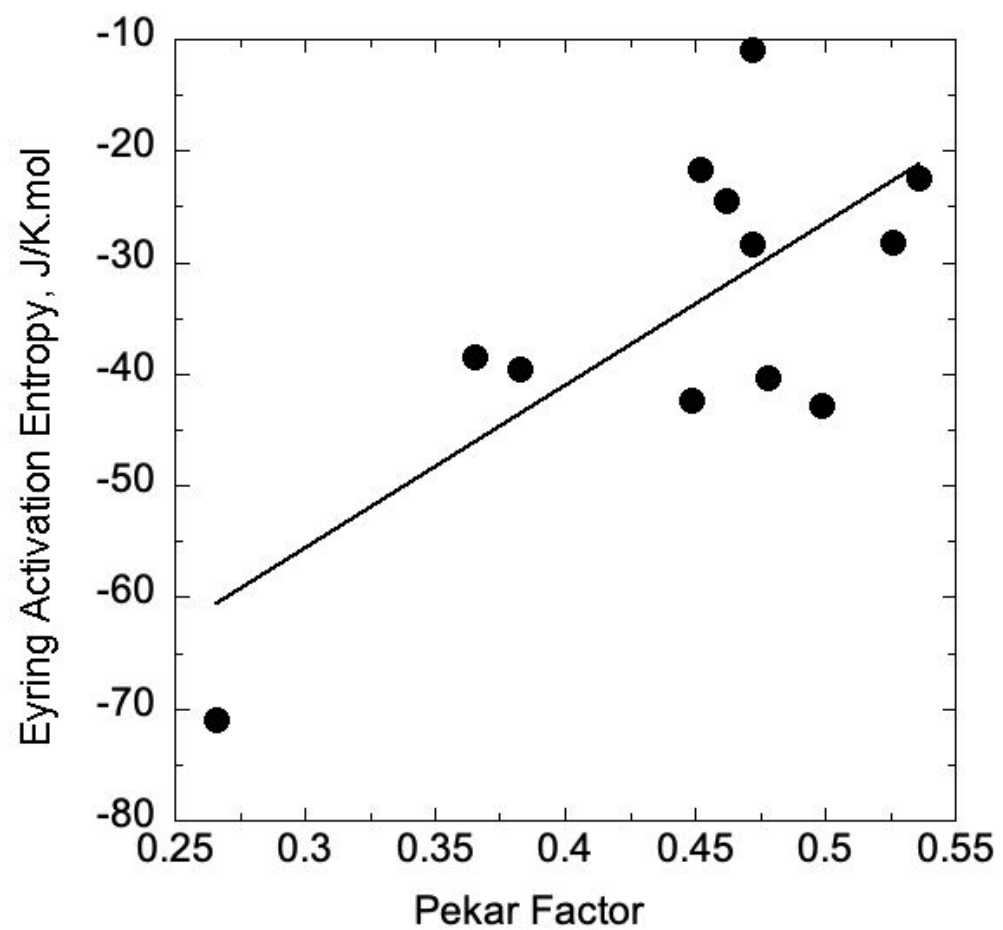

Figure S2. Plot of Eyring activation entropy for fragmentation of the pinacol radical cation as a function of the Pekar factor in various solvents. The correlation coefficient is 0.719 . 


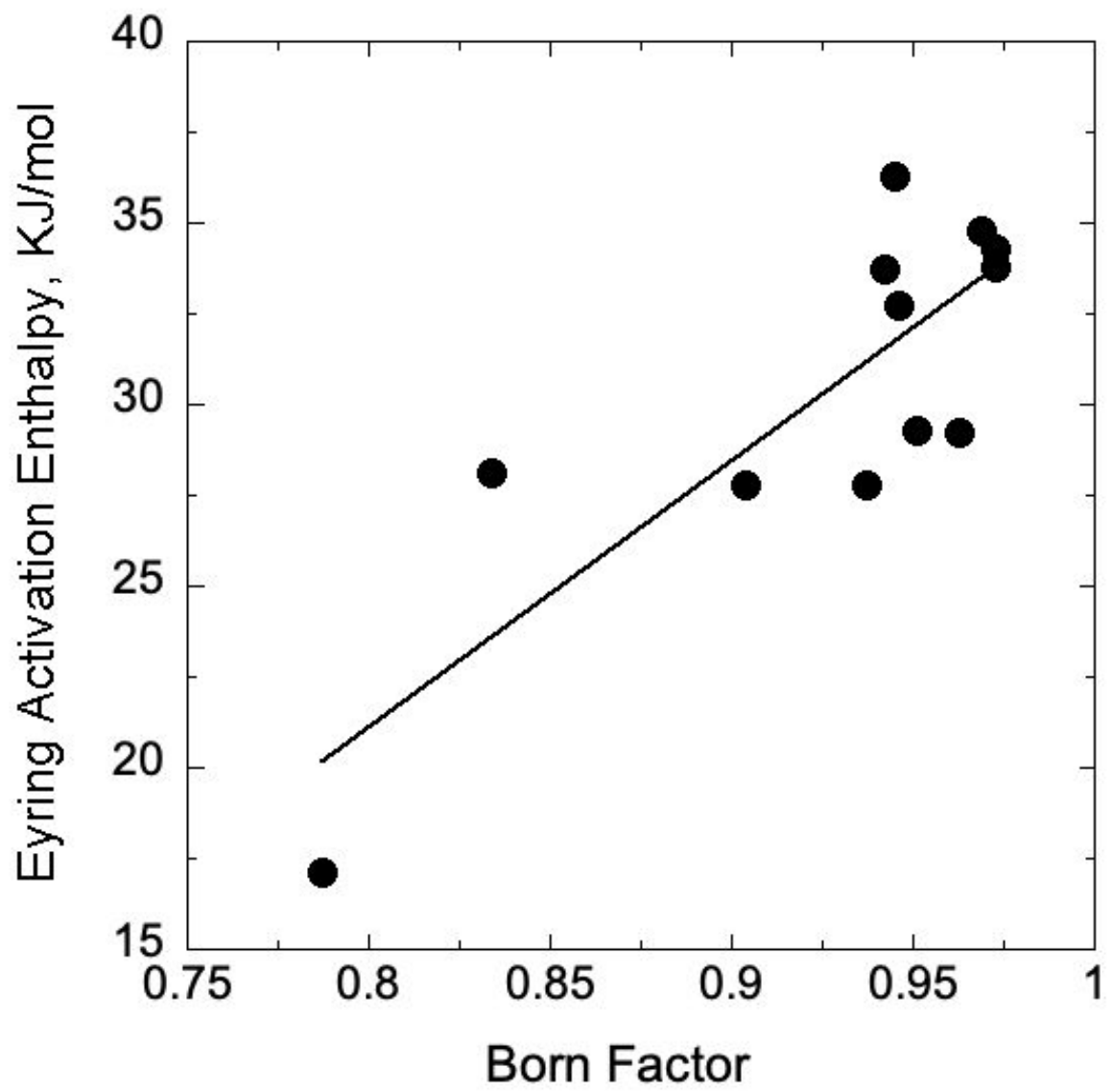

Figure S3. Plot of Eyring activation enthalpy for fragmentation of the pinacol radical cation as a function of the Born factor in various solvents. The correlation coefficient is 0.824 . 


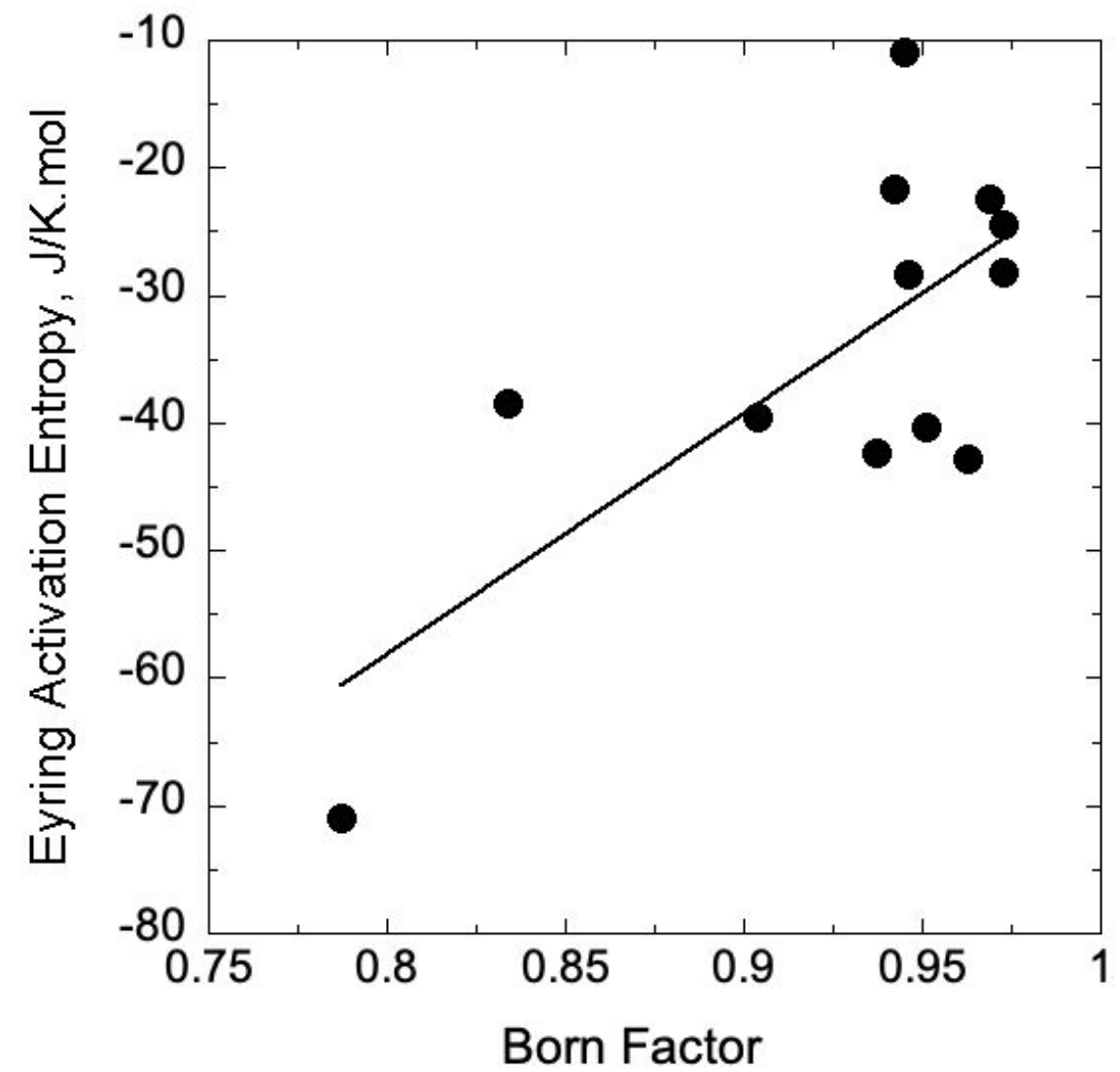

Figure S4. Plot of Eyring activation entropy for fragmentation of the pinacol radical cation as a function of the Born factor in various solvents. The correlation coefficient is 0.717 . 


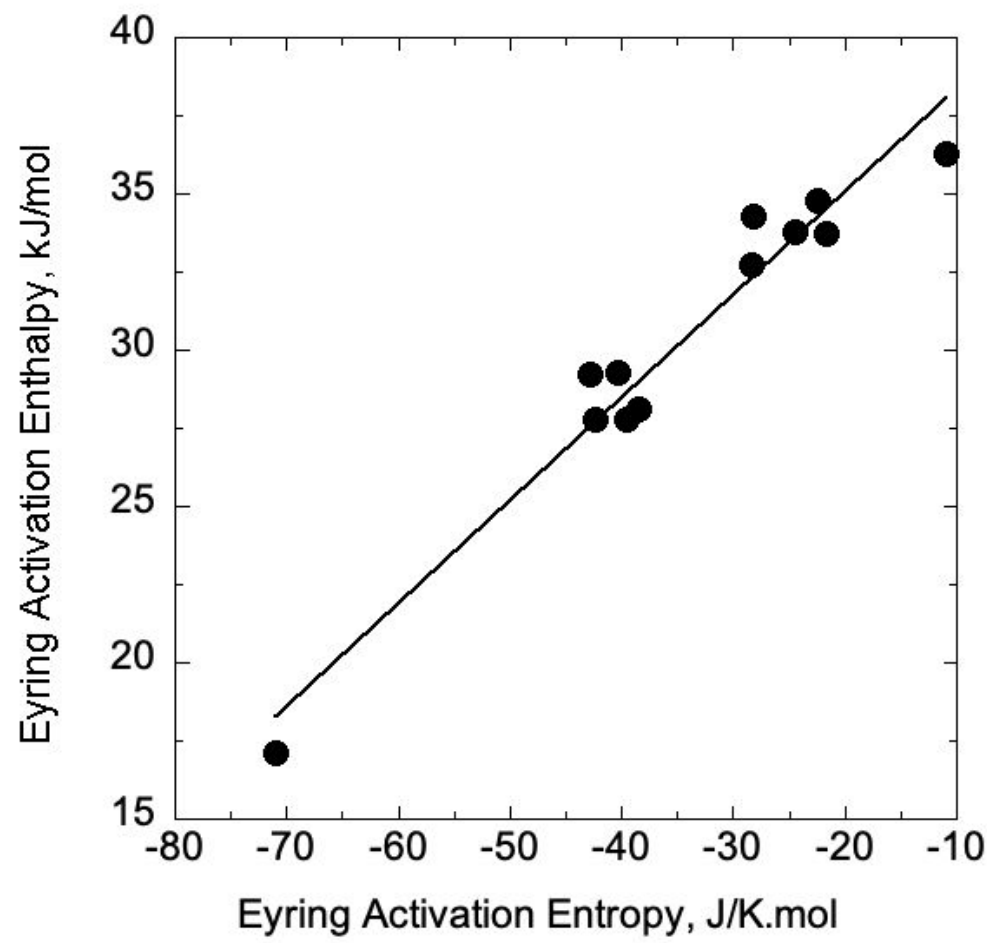

Figure S5. Plot of Eyring activation enthalpy versus Eyring activation entropy for fragmentation of the pinacol radical cation in various solvents. The correlation coefficient is 0.975 . 